\title{
Estudio Dinámico del Sistema Araña-Tela de Araña en condiciones de Resonancia
}

\author{
Jorge H. Alencastre ${ }^{(1)^{*}}$, Carlos Olarte ${ }^{(1)}$, Richard Rivera ${ }^{(1)}$ y José L. Muñoz ${ }^{(2)}$ \\ (1) Pontificia Universidad Católica del Perú, Departamento de Ingeniería, Lima 32-Perú \\ (e-mail: jalenca@pucp.edu.pe) \\ (2) Universidad Politécnica de Madrid, Departamento de Mecánica y Fabricación, ETSSI-España
}

Recibido Nov. 3, 2015; Aceptado Ene. 8, 2016; Versión final Mar. 8, 2016, Publicado Ago. 2016

\begin{abstract}
Resumen
Se ha estudiado el conjunto araña-tela de araña como un sistema dinámico, con la finalidad de obtener la respuesta de este sistema, cuando es sometido a condiciones de resonancia. La tela de araña es una estructura con una excelente combinación de resistencia, rigidez y tenacidad, óptima para transformar energía cinética en energía disipativa y así capturar a las presas. Sin embargo, hay condiciones ambientales externas que pueden llevar a esta estructura a condiciones de resonancia que podrían dañarla. De manera experimental se pudo comprobar que cuando ello ocurre, la araña es capaz de modificar la tensión de las sedas de la tela de araña, rigidizando la estructura para alejar al sistema de la zona de resonancia. La araña, mediante sus patas, censa la vibración en la tela de araña y actúa cuando las amplitudes aumentan significativamente y se mantienen por un largo periodo, cambiando así la tensión del sistema. Un modelo conceptual dinámico de un grado de libertad es una herramienta eficaz para replicar el comportamiento dinámico del conjunto araña-tela de araña en condiciones de resonancia.
\end{abstract}

Palabras clave: tela de araña; resonancia; vibraciones; dinámica estructural, análisis dinámico

\section{Dynamic Analysis of Spider-Spiderweb System under Resonant Conditions}

\begin{abstract}
The whole set spider-spiderweb is studied as a dynamic system in order to obtain the reaction of the system when subjected to conditions of resonance. A Spiderweb is a structure with a unique combination of strength, rigidity and tenacity, optimal to transform kinetic energy into deformation energy and capture prey. However, there are external environmental conditions that may lead this structure to resonance conditions that could damage it. It was experimentally shown that when this happens, the spider is able to modify the spiderweb silk tension, stiffening the structure to put the system away from resonance conditions. The spider senses, by its legs, the vibration in the spider web and acts when the amplitude increase significantly and remain for a long period, thus changing the system tension. A dynamic conceptual model whit one degree of freedom is an effective tool to represent the dynamic behavior of the set spider-spiderweb under resonance conditions.
\end{abstract}

Keywords: spiderweb; resonance; vibration; structural dynamics, dynamic analysis 


\section{INTRODUCCIÓN}

Las telas de araña que tejen las arañas de la especie Argiope argentata son estructuras que trabajan sometidas primordialmente a cargas de impacto. Para su buen funcionamiento dependen de una combinación óptima de las propiedades mecánicas de los componentes que la conforman (seda dragline y víscida), que les permiten absorber la energía de vuelo de las presas (Alencastre 2015; Ko 2004; Blackledge et al., 2006, Blackledge et al., 2005, Plaza et al., 2005, Plaza et al., 2008). Las arañas pueden producir hasta siete diferentes tipos de sedas, como la seda dragline (hilo de seguridad), víscida, espiral, capullo, etc. Cada una de estas sedas tiene diferentes propiedades mecánicas y funciones (Gosline, 1986; Vollrath, 2000, Gosline et al., 1999, Garrido et al., 2002, Garrido et al., 2002). La araña controla con precisión la secuencia de los aminoácidos de las proteínas que componen cada una de estas sedas (Vollrath, 1993). Las sedas de araña deben presentar una combinación insuperable de resistencia, deformación y tenacidad, para absorber la energía cinética de los insectos; pero, al mismo tiempo, la energía de retorno tiene que ser mínima, para evitar que la presa rebote de la tela de araña (Kelly et al., 2011).

La estructura de la tela de araña está compuesta por dos tipos de sedas: la seda dragline, segregada por la glándula ampulácea mayor (MA), con la cual se construyen los marcos y radios de la estructura; y la seda víscida, con la cual se construyen los hilos circunferenciales y que es producida por la glándula flageliforme (núcleo de la seda) y la glándula agregada que produce el recubrimiento pegajoso (Pérez Rigueiro, 2002, Montenegro, 2003). La combinación de la rigidez dada por la seda dragline y la gran capacidad de deformación de la seda víscida (pero ambas sedas con un valor aproximado $160 \mathrm{MJ} / \mathrm{m}^{3}$ de tenacidad) constituyen una estructura óptima para solicitaciones a cargas de impacto (Denny, 1976). Estudios recientes muestran que cuando la estructura es sometida a cargas de impacto de alta intensidad, tanto la rigidez como el amortiguamiento en los primeros ciclos de vibración transitoria presentan un comportamiento no lineal, zona donde las propiedades de las sedas tienen un alto grado de histéresis, lo que permite disipar la energía en estos primeros ciclos de vibración (Tietsch, 2015).

La tela de araña no solo permite una absorción rápida de la energía, sino que también suprime drásticamente la vibración, luego de ocurrido el impacto (Du et al., 2011). Estas vibraciones son usadas por las arañas para poder localizar y atacar a la presa. Barrows (1915) divulgó que, la araña de la especie Epeira sclopetaria, se posa en el centro de la tela de araña, para poder identificar la posición de impacto de las presas. Las arañas son capaces de discriminar entre los diferentes tipos de señal, como por ejemplo si es una presa o un artículo que cayó sobre su tela de araña (Parry, 1965). Sin embargo, existen factores climáticos como el viento, que pueden llevar al sistema araña - tela de araña a situaciones de vibración en condiciones de resonancia que producirían daño en la estructura (Alencastre, 2007, Alencastre, 2015). Por ello, se planteó realizar una investigación experimental-analítica, abordando a todo el conjunto araña-tela de araña como un sistema dinámico con el fin de observar la reacción de este sistema cuando es sometido a solicitaciones en condiciones de resonancia. A partir de los resultados obtenidos se puede plantear aplicaciones en diferentes campos en los que se presentan sistemas que puedan entrar en resonancia.

\section{METODOLOGÍA}

En la investigación se utilizó el concepto de vibración forzada como herramienta para determinar las propiedades de rigidez y amortiguamiento de los sistemas araña-tela de araña y al mismo tiempo describir el comportamiento dinámico de los sistemas, con modelos conceptuales de sistemas de un grado de libertad.

Se utilizaron dos técnicas diferentes para la excitación de los sistemas. En el primer procedimiento se utilizó un parlante para excitar al sistema araña-tela de araña. La frecuencia y potencia del sonido emitido por el parlante se controlaron desde una computadora. Se utilizó, también, un micrófono omnidireccional para medir la presión sonora de excitación. y con el velocímetro láser, gracias a un elemento reflectivo adherido a la tela de araña, se registró la velocidad a la cual vibraba el sistema. Los resultados obtenidos con esta técnica no fueron suficientemente coherentes debido a que los niveles de presión sonora logrados producían desplazamientos muy pequeños $(<0,5 \mathrm{~mm})$, que se confundían con aquellos inducidos por el ruido del ambiente; por ende, se estimó conveniente experimentar con otra técnica. La segunda técnica con la que se realizaron los estudios experimentales de esta investigación, consistió en una mesa vibratoria capaz de proporcionar un movimiento armónico, generando así la excitación de los sistemas, mediante el movimiento armónico del marco soporte que estaba fijado a la mesa vibratoria. La figura 1a muestra el montaje del marco y la mesa vibratoria. La frecuencia de excitación de la mesa fue controlada con un regulador; con el velocímetro láser y con un reflectivo adherido a la tela de araña se registró la velocidad de las masas (las arañas); con el acelerómetro se registró la aceleración de la mesa vibratoria, de modo que, se registró en todo momento el desplazamiento total de las masa (en este caso las arañas) en función del tiempo. La figura $1 \mathrm{~b}$ muestra el esquema técnico de este segundo método experimental.

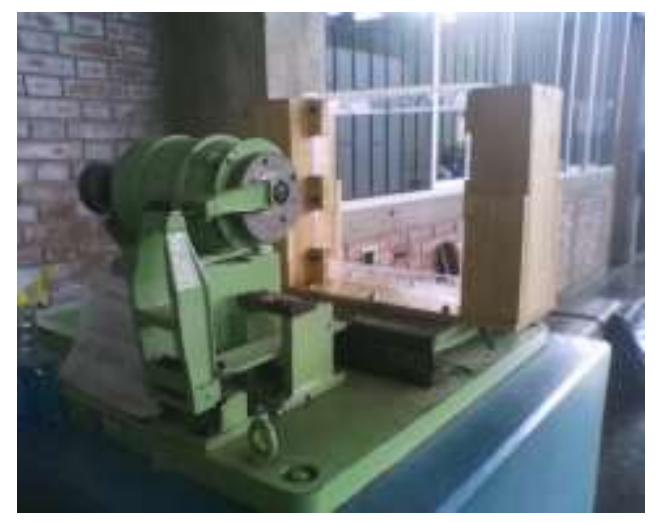

Fig. 1a: Imagen del montaje experimental

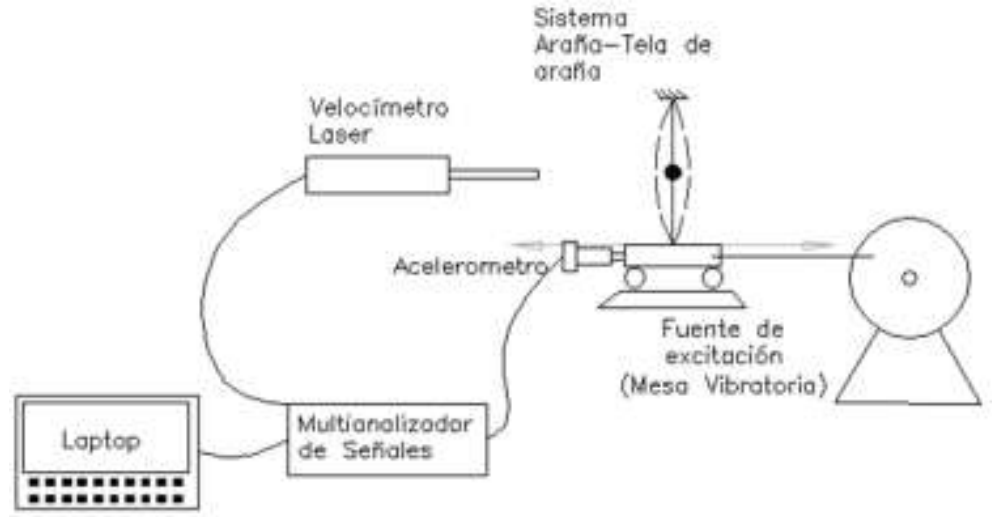

Fig.1b: Esquema técnico de las pruebas experimentales 
Se trabajó con cinco arañas de la especie Argiope argentata, que tejieron sus telas en marcos cuadrados de acrílico de $300 \mathrm{~mm} \times 300 \mathrm{~mm}$ de lado, como se puede apreciar en la figura 1a. Paralelamente se desarrolló un modelo analítico, como un sistema dinámico de un grado de libertad, sometido a una excitación de base, con la finalidad de describir el comportamiento de los sistemas y así poder evaluar los resultados experimentales. El desarrollo del modelo conceptual se puede apreciar en la figura 2.
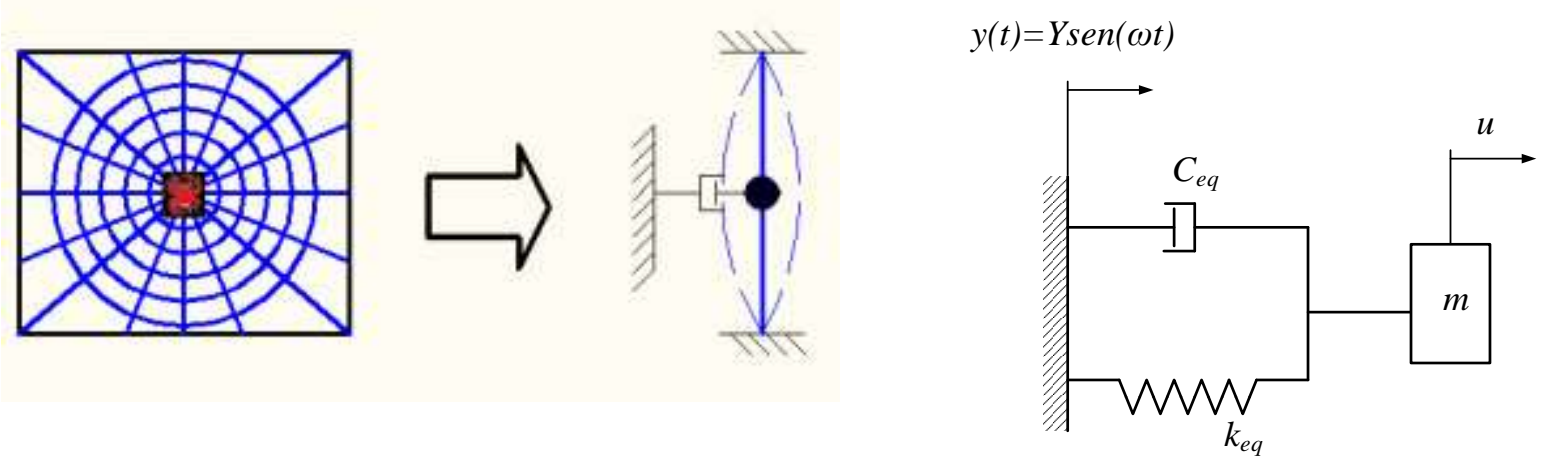

Fig. 2: Modelo conceptual del sistema araña-tela de araña, con excitación de base

La ecuación diferencial de movimiento linearizado del sistema, mostrado en la figura 2, viene dada por la siguiente formulación.

$$
\ddot{z}+\frac{C_{e q}}{m} \dot{z}+\frac{k_{e q}}{m} z=Y \omega^{2} \operatorname{sen}(\omega t) \quad ; z=u-y
$$

En la ecuación anterior $m$ representa la masa de cada araña, Ceq es el amortiguamiento equivalente global (tanto del aire y la intrínseca a la tela de araña), keq es la rigidez equivalente de la tela de araña, $\omega$ es la frecuencia de excitación de la mesa vibratoria, $Y$ es la amplitud de vibración de la mesa y $z, u$, y son coordenadas para describir el movimiento del sistema.

\section{RESULTADOS Y DISCUSIÓN}

Para dar inicio a las pruebas experimentales se inició con el pesaje de las arañas, que se realizó en una balanza electrónica de precisión de una sensibilidad de 0,0001g. Cabe mencionar que no se tomó en cuenta la masa de la tela de araña, siendo despreciable con respecto a la masa de la araña (Alencastre 2007). En la tabla 1 se muestran los pesos de las diferentes arañas, que representarán las masas en el modelo conceptual.

Tabla 1: Masa de las arañas

\begin{tabular}{|c|c|c|c|c|c|}
\hline \multirow{2}{*}{} & \multicolumn{5}{|c|}{ Masa de las arañas } \\
\cline { 2 - 6 } & Araña 1 & Araña 2 & Araña 3 & Araña 4 & Araña 5 \\
\hline Masa (g) & 0,2014 & 0,6003 & 0,2716 & 0,5288 & 0,2937 \\
\hline
\end{tabular}

Los sistemas de las arañas 1 y 2 fueron excitados mediante la primera técnica experimental, mientras que los sistemas de las arañas 3 a 5 se excitaron con la segunda técnica experimental de la mesa vibratoria. Mediante pruebas de run-up y cost-down se hizo un barrido inicial de frecuencias para cada uno de los sistemas (araña tela de araña), esto con la finalidad de determinar las frecuencias naturales de los mismos. Promediando las cinco pruebas realizadas a cada sistema, se determinó que estos son de baja frecuencia natural (flexibles). El rango de frecuencias naturales de los sistemas experimentados oscila entre los 3 y $5 \mathrm{~Hz}$. Los únicos dos parámetros conocidos son la fuente de excitación y la masa de las arañas. El valor de rigidez equivalente y el amortiguamiento equivalente se obtienen del barrido de frecuencias de cada sistema, a través de la frecuencia natural y la brusquedad de la resonancia. En la tabla 2 se pueden apreciar los valores promedio de cada uno de los sistemas.

Tabla 2: Propiedades de los Sistemas 3-5

\begin{tabular}{|l|c|c|c|}
\hline & Sistema 3 & Sistema 4 & Sistema 5 \\
\hline Frecuencia natural $(\mathrm{Hz})$ & 2,813 & 3,1 & 3,3 \\
\hline Semilongitud del marco soporte L $(\mathrm{mm})$ & 150 & 150 & 150 \\
\hline Masa de la araña $(\mathrm{gr})$ & 0,2716 & 0,5288 & 0,2937 \\
\hline Rigidez equivalente $(\mathrm{N})$ antes de la resonancia & 0,0064 & 0,0141 & 0,0099 \\
\hline Rigidez equivalente(N) post-resonancia & 0,00992 & 0,021 & 0,0169 \\
\hline Coeficiente de Amortiguamiento Equivalente $(\mathrm{Ns} / \mathrm{m})$ & 0,0001707 & 0,00135 & 0,0089 \\
\hline Factor de Amortiguamiento $\xi$ (adimensional) & 0,0178 & 0,0677 & 0,0716 \\
\hline
\end{tabular}

Una vez determinada la frecuencia natural de los sistemas, se realizaron las pruebas respectivas, llevando a cada uno de los sistemas al rango de resonancia. La figura 3 muestra la variación tanto de la amplitud como de la frecuencia en función del tiempo de uno de los ensayos realizados a la araña 5. Adicionalmente, se observa el tiempo que se mantuvo al sistema en la región de resonancia. 

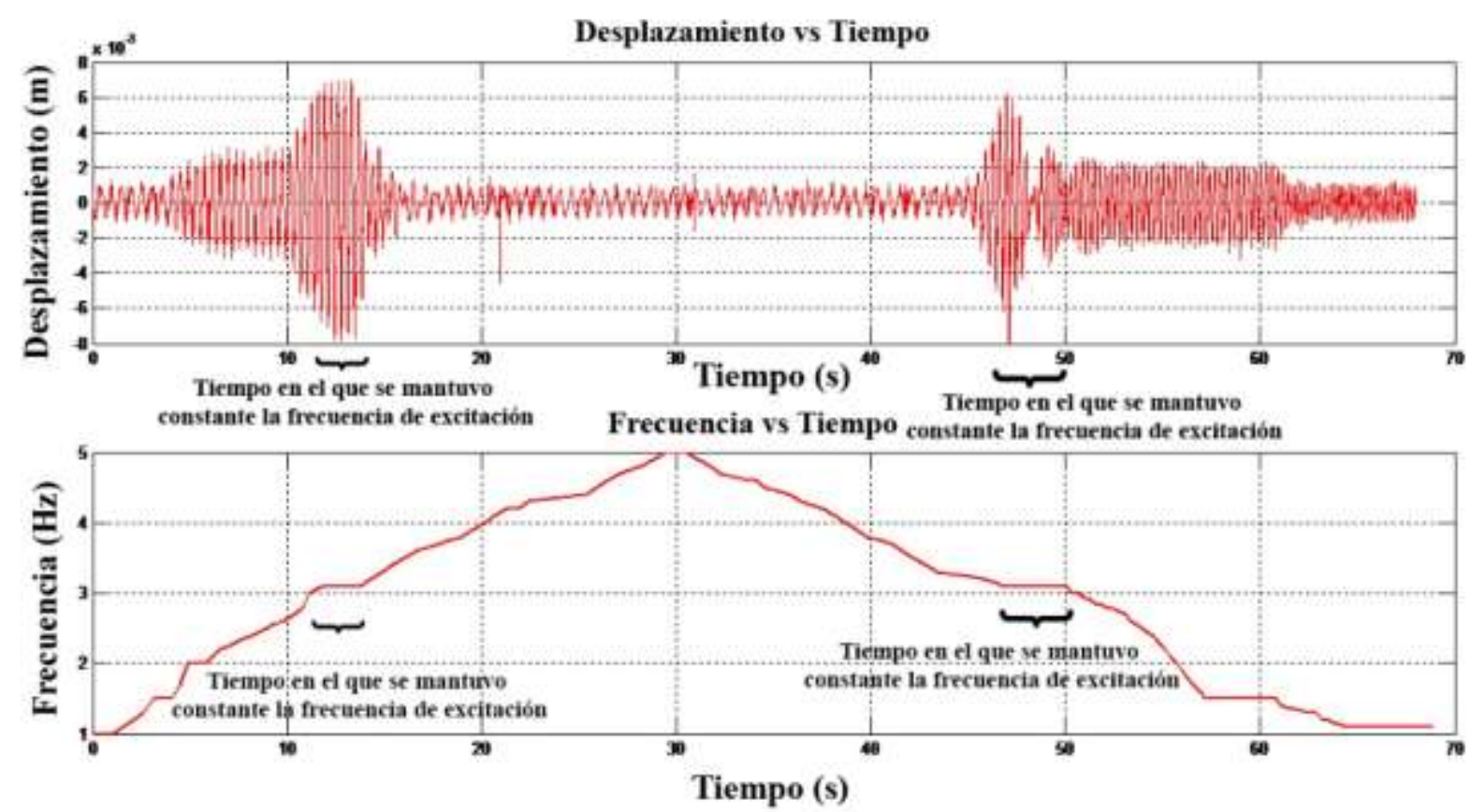

Fig. 3: Desplazamiento y frecuencia de excitación en función del tiempo

La figura 4 muestra una ampliación de la zona de resonancia, donde se mantuvo la frecuencia de excitación constante. En ella se pueden observar tres etapas: la etapa 1 describe el comportamiento durante la resonancia; la etapa 2, durante el cambio de la tensión; y la etapa 3, durante la postresonancia.

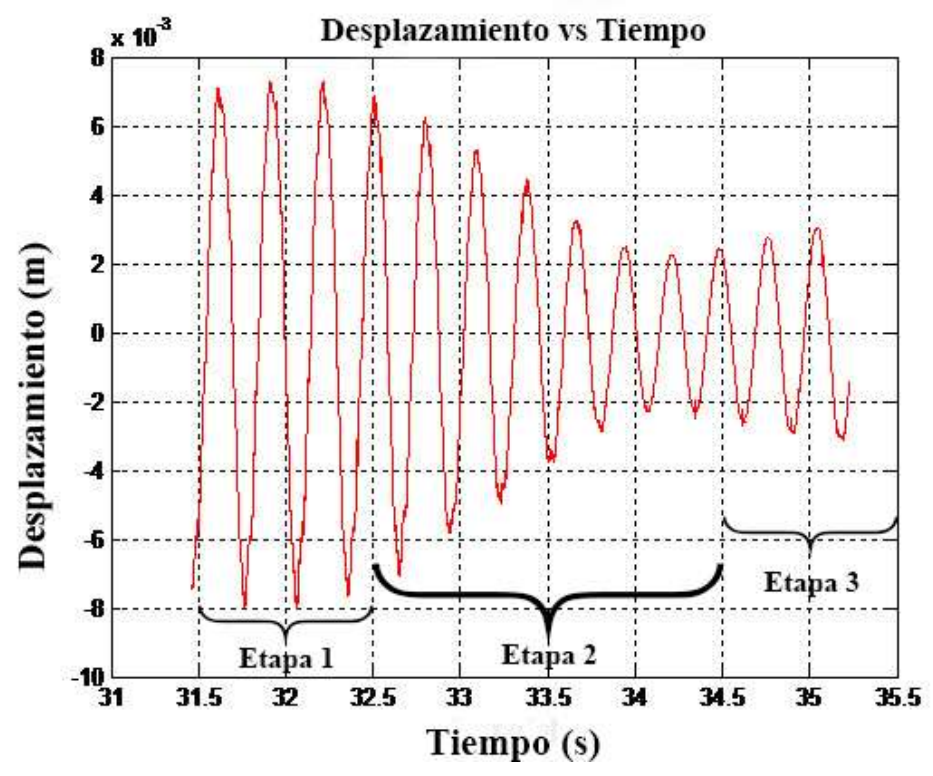

Fig.4: Desplazamientos durante la etapa de resonancia

Los valores de rigidez y amortiguamiento equivalentes, obtenidos del barrido de frecuencias conjuntamente con los valores de las masas de las arañas, así como la frecuencia y amplitud de la mesa vibratoria, se introdujeron en la ecuación 1 para obtener la respuesta del modelo analítico. Dicho modelo replica correctamente el comportamiento del sistema durante la resonancia como se aprecia en la figura $5 a$ y en la etapa de postresonancia, como muestra la figura $5 b$, para el sistema araña 5.

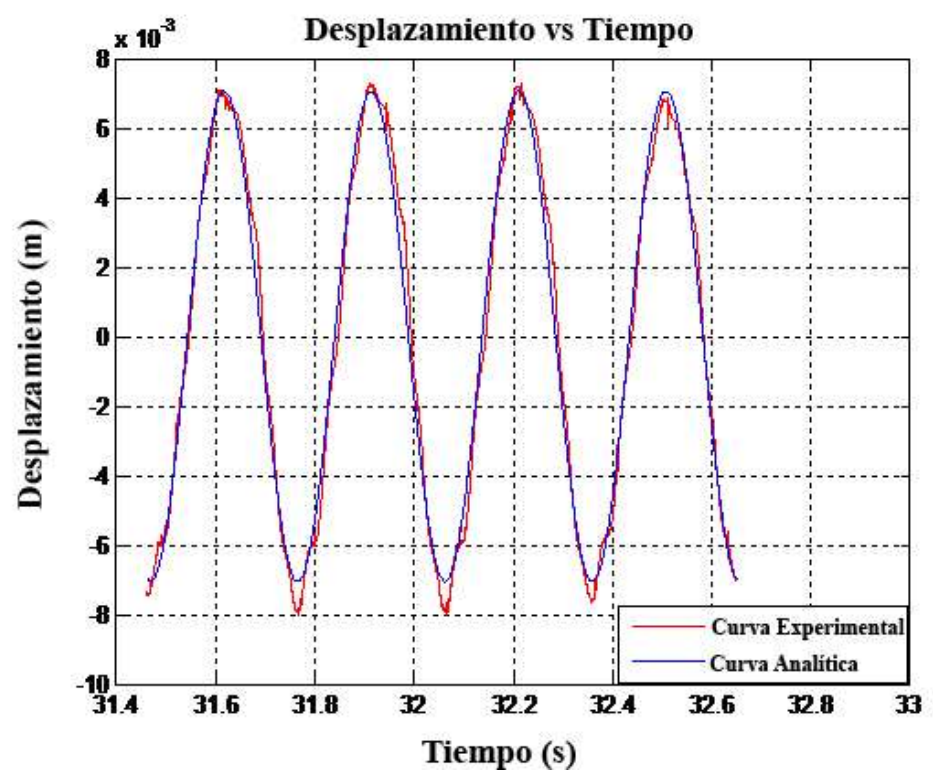

Fig. 5a: Desplazamiento durante la resonancia 


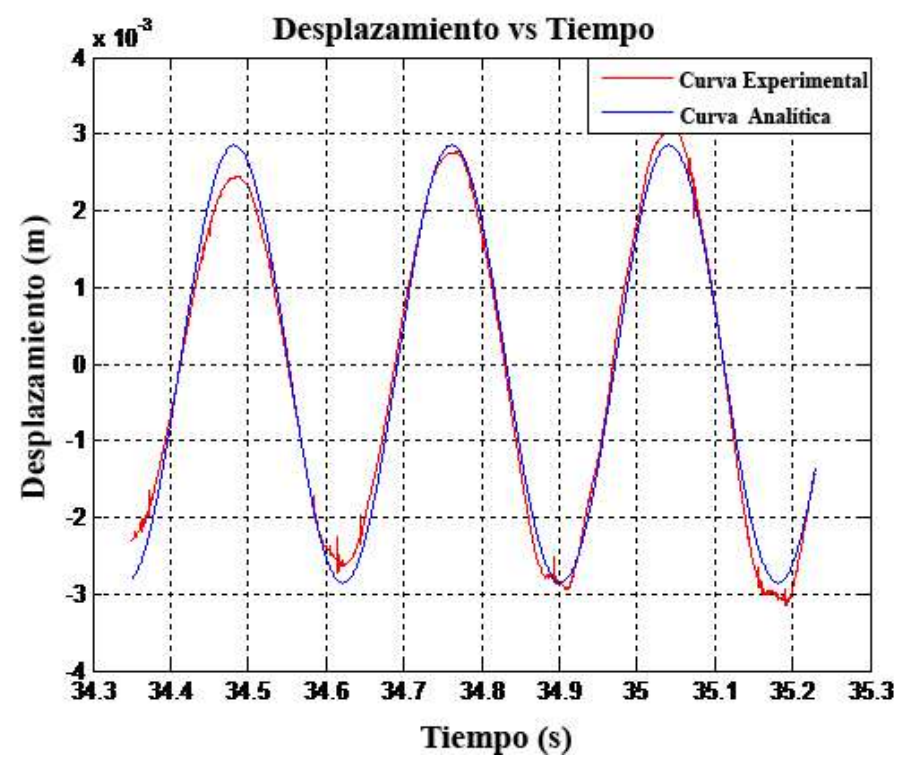

Fig. 5b: Desplazamiento post-resonancia

Sin embargo, en la etapa en la cual la araña cambia la tensión del sistema, el modelo conceptual no representa correctamente el comportamiento dinámico del sistema. El único parámetro que puede variar es la rigidez equivalente, puesto que la masa de la araña no cambia y el valor del amortiguamiento equivalente no es representativo, por ser muy pequeño. La figura 4 muestra que, durante ese periodo, dicho parámetro varía con el tiempo. Para ello se realiza un ajuste de este parámetro mediante una aproximación armónica de la siguiente forma:

$$
\begin{aligned}
k_{e q}(t)= & \left(Y C_{e q} \omega \cos (\omega t)+a_{1} b_{1}^{2} m \operatorname{sen}\left(c_{1}+b_{1} t\right)+a_{2} b_{2}^{2} m \operatorname{sen}\left(c_{2}+b_{2} t\right) \ldots\right. \\
& \left.-a_{1} b_{1} C_{e q} \cos \left(c_{1}+b_{1} t\right)-a_{2} b_{2} C_{e q} \cos \left(c_{2}+b_{2} t\right)\right) / \ldots \\
& \left(a_{1} \operatorname{sen}\left(c_{1}+b_{1} t\right)+a_{1} \operatorname{sen}\left(c_{1}+b_{1} t\right)-Y \operatorname{sen}(\omega t)\right)
\end{aligned}
$$

En la ecuación anterior los coeficientes $a_{1}, a_{2}, b_{1}, b_{2}, c_{1}$ y c2 son coeficientes de aproximación que se obtienen de un programa Matlab R2010a. La ecuación (1) es modificada a la siguiente expresión:

$$
\ddot{z}+\frac{C_{e q}}{m} \dot{z}+\frac{k_{e q}(t)}{m} z=Y \omega^{2} \operatorname{sen}(\omega t)
$$

Introduciendo los valores de rigidez equivalente obtenidos en la igualdad 2 en la ecuación diferencial modificada 3 , se obtienen resultados analíticos que describen correctamente el comportamiento del sistema durante la etapa del cambio de rigidez. La figura 6 representa la respuesta tanto del modelo analítico como los resultados experimentales para el sistema 5. Se observa una clara coincidencia entre ambos.

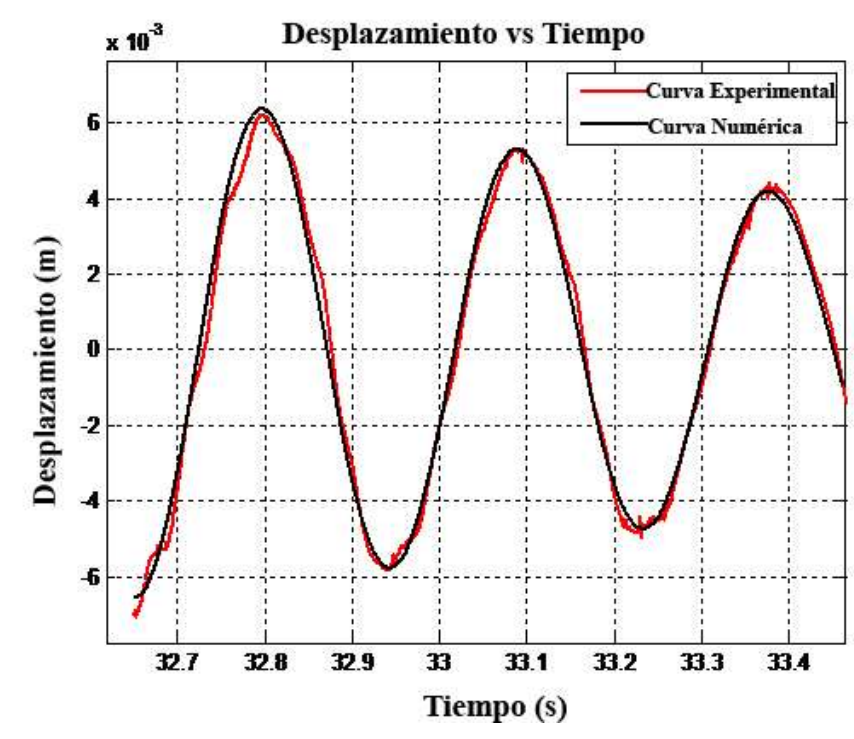

Fig. 6: Desplazamientos durante la etapa del cambio de la tensión

Los resultados permiten verificar que la hipótesis planteada, que la araña tiene la capacidad instintiva de variar la tensión de las cuerdas radiales y así cambiar la rigidez equivalente y por ende su frecuencia natural, cuando el sistema es excitado a su frecuencia de resonancia, se cumple. Durante las experimentaciones se pudo observar que las arañas, con la ayuda de sus patas, realizaban acciones sobre las cuerdas radiales que conllevan a cambiar la tensión total del sistema y, de esta forma, también su frecuencia natural. Se determinó que la tensión equivalente sufre un aumento significativo (como se puede apreciar en la tabla 2), y varía para cada sistema araña - tela de araña. Los valores experimentales muestran un aumento de este parámetro, entre el $50 \%$ y $70 \%$, lo que implica una reducción de las amplitudes de vibración hasta en un $50 \%$. Durante el cambio de tensión existe un intervalo de tiempo en que la tensión y, por consiguiente, la constante de rigidez no 
dependen solo de los parámetros vibratorios, sino también de la forma en que cada araña responde frente a la resonancia. Los valores del factor de amortiguamiento equivalente son pequeños (del orden de 0,07 ) y solo se consideró un valor global, debido a que el amortiguamiento propio del material es muy pequeño- en comparación con el amortiguamiento, por ejemplo, del aire, siendo el parámetro de la rigidez equivalente el más significativo e influyente en el sistema. Los valores de frecuencias naturales obtenidos en esta investigación se encuentran dentro del rango de frecuencias obtenidas en otras investigaciones. Luego de evaluar el comportamiento de los sistemas araña-tela de araña en condiciones de resonancia, es posible copiar la capacidad sensorial que tiene la araña para cambiar la rigidez equivalente de la tela de araña para salir del rango de resonancia.

\section{CONCLUSIONES}

La principal conclusión de este trabajo es que un modelo conceptual dinámico de un grado de libertad es una herramienta eficaz para replicar el comportamiento dinámico del conjunto araña-tela de araña en condiciones de resonancia.

\section{REFERENCIAS}

Alencastre, J., Mago, C. y Rivera R., Determination of energy dissipation of a spider silk structure under impulsive loading, Frontier of Mechanical Engineering, 1-5 (2015)

Alencastre, J., Vera Mechán, J., Muñoz Sanz, J.L., Echavarri, J., Characterization of Dynamic Parameters of a Structure Made of Spider Silk Dragline, International Journal of Applied Science and Technology, Vol. 5, No. 5; October (2015)

Alencastre Miranda, J., Vera Mechán, J. Propiedades dinámicas de la tela de araña orbicular. $8^{\circ}$ Congreso Iberoamericano de Ingeniería Mecánica. 23-25 Octubre (2007)

Barrows, W. M., The reactions of an orb-weaving spider, Epeira sclopetaria Clerck, to rhythmic vibrations of its web. Biological Bulletin, 29(5), 316-332 (1915)

Blackledge, T. A., y Hayashi, C. Y., Unraveling the mechanical properties of composite silk threads spun by cribellate orb-weaving spiders. The Journal of experimental biology, 209(16), 3131-3140 (2006)

Blackledge, T. A., Swindeman, J. E., y Hayashi, C. Y., Quasistatic and continuous dynamic characterization of the mechanical properties of silk from the cobweb of the black widow spider Latrodectus hesperus. Journal of Experimental Biology, 208(10), 1937-1949 (2005)

Denny, M., The physical properties of spider's silk and their role in the design of orb-webs, J. exp. Biol, 65(2), 483-506 (1976)

Du, N., Yang, Z., Liu, X. Y., Li, Y., e Xu, H. Y., Structural Origin of the Strain-Hardening of Spider Silk. Advanced Functional Materials, 21(4), $772-778$ (2011)

Garrido, M. A., Elices, M., Viney, C. Pérez-Rigueiro, J., The variability and interdependence of spider drag-line tensile properties. Polymer 43, 4495-4502. (2002)

Garrido, M. A., Elices, M., Viney, C. Pérez-Rigueiro, J., Active control of spider silk strength: comparison of dragline spun on vertical and horizontal surfaces. Polymer 43 1537-1540 (2002)

Gosline, J. M., DeMont, M. E., y Denny, M. W., The structure and properties of spider silk. Endeavour, 10(1), 3743 (1986)

Gosline, J., Guerette, P., Ortelepp, C., and Savage, K., The Mechanical Design of Spider Silks: From Fibroin Sequence to Mechanical Function, The Journal of Experimental Biology, 202, 3295-3303. (1999)

Kelly, S. P., Sensenig, A., Lorentz, K. A., y Blackledge, T. A., Damping capacity is evolutionarily conserved in the radial silk of orb-weaving spiders. Zoology, 114(4), 233-238 (2011)

Ko, Frank, Jovicic Jovan, Modelling of mechanical properties and structural design of spider web. Biomacromolecules 5, 780-785 (2004)

Montenegro, Rivelino. La asombrosa telaraña. Ciencia de los orígenes N.66, (2003)

Parry, D. A., The signal generated by an insect in a spider's web. Journal of Experimental Biology, 43(1), 185$192(1965)$

Pérez Rigueiro,J. Elices,M., y Guinea, G.V. Estrategias de la naturaleza en el diseño de materiales: La Seda de Araña Biblioteca en línea del Grupo Ibérico de Aracnología, Sociedad Entomológica Aragonesa, (2002)

Plaza, G.R., Pons, A., Elices,M., Pérez-Rigueiro, J., Guinea, G.V. Absorción, disipación de energía mecánica y rotura de la seda víscida de araña. Anales de Mecánica de la Fractura, vol. 22, 258-263 (2005)

Plaza, G.R., Musa, A., Pérez-Rigueiro, J., Pons,A., Perea, G.B., Elices,M., Guinea, G.V. Comparación de la resistencia mecánica y disipación de energía de la seda de seguridad y de la seda víscida de araña. Anales de Mecánica de la Fractura 25, Vol. 1, 41-46 (2008)

Tietsch, V., Alencastre,J., Witte, H., Torres, F.G. Exploring the Shock Response of Spider Webs, Journal of the Mechanical Behavior of Biomedical Materials, elsevier_JMBBM_1680, (2015)

Vollrath, F., General Properties of Some Spider Silks, in Kaplan, D., Adams, W., Farmer, B., Viney, C., Eds. Silk Polymers, American Chemical Society, (1993)

Vollrath, F., Strength and Structure of Spider's Silks, Molecular Biotechnology, 74, 67-83, (2000) 